\title{
Correction to: Atorvastatin-loaded solid lipid nanoparticles as eye drops: proposed treatment option for age-related macular degeneration (AMD)
}

\author{
Monika Yadav ${ }^{1} \cdot$ Nicola Schiavone $^{2} \cdot$ Ana Guzman-Aranguez $^{3} \cdot$ Fabrizio Giansanti $^{2} \cdot$ Laura Papucci $^{2}$. \\ Maria J. Perez de Lara $^{3} \cdot$ Mandeep Singh ${ }^{1} \cdot$ Indu Pal Kaur ${ }^{1}$ \\ Published online: 3 May 2020 \\ (C) Controlled Release Society 2020
}

\section{Correction to: Drug Delivery and Translational Research https://doi.org/10.1007/s13346-020-00733-4}

In the original version of this article Ana Guzman-Aranguez's name appeared incorrect. It is correct as reflected here. The original article has been updated.

The online version of the original article can be found at https://oi.org/ 10.1007/s13346-020-00733-4

\footnotetext{
Indu Pal Kaur

dripkuips@gmail.com; indupalkaur@yahoo.com

1 Department of Pharmaceutics, University Institute of Pharmaceutical Sciences, Panjab University, Chandigarh 160014, India

2 Department of Experimental and Clinical Biomedical Sciences, Section of Experimental Pathology and Oncology, Viale Morgagni 50, 50134 Florence, Italy

3 Facultad de Optica y Optometria, Dpto. Bioquimica y Biologia, Molecular IV, Universidad Complutense de Madrid, C/Arcos de Jalon 118, 28037 Madrid, Spain
} 